\title{
Immediate changes in biochemical markers of bone turnover and circulating interleukin- 6 after parathyroidectomy for primary hyperparathyroidism
}

\author{
Chun-Yuan Guo ${ }^{1}$, Philip A Holland ${ }^{2}$, Brendan F Jackson ${ }^{1}$, Rosemary A Hannon ${ }^{1}$, Angela Rogers ${ }^{1}$, \\ Barnard J Harrison $^{2}$ and Richard Eastell ${ }^{1}$ \\ ${ }^{1}$ Department of Human Metabolism and Clinical Biochemistry, University of Sheffield and ${ }^{2}$ Department of Surgery, Northern General Hospital \\ NHS Trust, Sheffield S5 7 AU, UK \\ (Correspondence should be addressed to R Eastell, Department of Human Metabolism \& Clinical Biochemistry, Clinical Sciences Centre, \\ Northern General Hospital, Sheffield S5 7AU, UK; Email: r.eastell@sheffield.ac.uk)
}

\begin{abstract}
Objective: The time course of the immediate change in bone turnover after parathyroidectomy (PTX) for primary hyperparathyroidism (PHPT) is not clear. It is uncertain whether circulating interleukin-6 (IL-6) plays a role in mediating the acute withdrawal of the effects of parathyroid hormone (PTH) on bone turnover after PTX. The aims of this study were to determine the time course of immediate changes in biochemical markers of bone turnover after PTX and whether circulating IL-6 is involved in the immediate changes of bone turnover after PTX.

Design and Methods: IL-6 and bone turnover markers were measured in eight women (aged $55 \pm 11$ years, mean \pm S.D.) with PHTP at baseline and at 1-2 h, and 1, 2, 5, 7 and 12 days after PTX. We compared the results with those from eight individually matched women (healthy controls) and five subjects undergoing major surgery (surgical controls).

Results: At baseline, serum levels of IL- 6 and bone turnover markers were higher in PHPT than those in healthy controls $(P<0.05)$. Serum levels of procollagen propeptides increased by 22 and $27 \%$ at days 2 and 5 , respectively, compared with baseline $(P<0.05)$. Serum tartrate-resistant acid phosphatase decreased by 2 days after PTX, and urinary collagen crosslinks decreased significantly by $21-41 \%$ within $24 \mathrm{~h}(\mathrm{P}<0.05)$. Serum IL-6 levels increased immediately in both PHPT and surgical controls at postoperative follow-up (repeated measures ANOVA).

Conclusions: (1) PTX decreases bone resorption immediately and (2) circulating IL-6 is not involved in the changes in bone turnover immediately after PTX.

European Journal of Endocrinology 142 451-459
\end{abstract}

\section{Introduction}

The immediate effects of parathyroid hormone (PTH) on bone could be examined by injecting PTH or by studying changes immediately after parathyroidectomy (PTX). It has been reported that PTH infusion increases urinary pyridinoline (Pyd), deoxypyridinoline (Dpd) (1) and hydroxyproline (OHP) (1-4), and decreases serum procollagen type I C-terminal propeptide (PICP) $(5,6)$ and osteocalcin (bone Gla-protein, BGP) (4). Bone turnover is increased in patients with primary hyperparathyroidism (PHPT). The increased bone turnover may lead to osteopenia which is the most common skeletal abnormality in PHPT even though it is debated whether mild to moderate PHPT causes osteopenia (7-9). PTX may restore bone turnover to normal. However, the time course of the restoration in bone turnover immediately after PTX is unclear.

It is generally agreed that PTH does not act directly on osteoclasts to stimulate bone resorption. PTH increases the number and activity of osteoclasts by acting on osteoblasts which release cytokines such as interleukin-6 (IL-6) (10). Recently, a study reported that serum circulating levels of IL-6 and IL-6 soluble receptors were increased significantly in untreated patients with PHPT and fell into the normal range after surgery (11). It is unknown whether the changes in biochemical markers of bone resorption are explained by the changes in circulating IL- 6 immediately after surgery. The aims of this study were to determine the time course of immediate changes in biochemical markers of bone turnover after PTX and whether circulating IL- 6 is involved in the immediate changes in bone turnover after PTX.

\section{Subjects}

Eight women (aged $55 \pm 11$ years, mean \pm S.D.) with PHPT were recruited from the Department of Surgery 
at the Northern General Hospital, Sheffield. Five of the eight patients were postmenopausal. These women were $2-21$ years postmenopause ( $8 \pm 5$ years). Patients had no other diseases known to affect bone metabolism or history of fracture in the preceding 2 years. Patients were not taking any medication known to affect bone metabolism, such as oestrogen, glucocorticoids, anticonvulsants, vitamin D or calcium supplements. The diagnosis of PHPT was based on preoperative clinical and laboratory criteria and postoperative pathology. Serum Ca levels ranged from 2.70 to $2.95 \mathrm{mmol} / \mathrm{l}$ $(2.80 \pm 0.12 \mathrm{mmol} / \mathrm{l})$ and PTH levels ranged from 34 to $134 \mathrm{pg} / \mathrm{ml}(110 \pm 55 \mathrm{pg} / \mathrm{ml})$. Serum PTH levels were above the upper limit of reference interval $(63 \mathrm{pg} / \mathrm{ml})$ in seven of the eight patients. Eight healthy controls individually matched for age and sex were used for comparison to baseline values. Five patients (four women and one man, aged 33-76 years) who underwent cholecystectomy, sigmoid colectomy or hemicolectomy were recruited as surgical controls. All control subjects were free from diseases and were not taking any medication known to affect bone metabolism. All participants gave written informed consent, and the study was approved by the North Sheffield Local Research Ethics Committee.

\section{Methods}

Serum levels of IL-6, BGP, bone alkaline phosphatase (BAP), PICP, N-terminal of type I procollagen (PINP) and tartrate-resistant acid phosphatase (TRAP) were measured in patients with PHPT at baseline and at 1$2 \mathrm{~h}$, and 1, 2, 5, 7 and 12 days after PTX. Serum levels of biochemical markers of bone turnover and IL- 6 were also measured in the five surgical controls at baseline and 2, 7 and 14 days after operation. Biochemical markers of bone turnover and IL- 6 were measured only at baseline in the eight age-matched women as healthy controls. Baseline samples (preoperative) were collected twice from five of the eight patients with PHPT. In these five patients, baseline values were taken as the mean of the two baseline collections. All blood samples, except those taken $1-2 \mathrm{~h}$ after PTX, were taken in the fasting state. All blood samples were centrifuged for $30 \mathrm{~min}$ at $2000 \mathrm{~g}$ after they were taken and the serum was separated and stored at $-70^{\circ} \mathrm{C}$. Serum samples were measured at the end of the study in duplicate in a single analytical batch.

Two hour urine samples (second morning void) were collected in PHPT group and healthy control group in this study. Urinary levels of total Pyd, total Dpd and cross-linked N-telopeptide of type I collagen (NTx) were measured at the same times as measured for blood samples except at $1-2 \mathrm{~h}$ after PTX.

Serum IL-6 was measured by ELISA (Quantikine, R\&D Systems, Inc., Minneapolis, USA). Serum BGP was measured by ELISA (NovoCalcin, Metra Biosystems, Inc., Mountain View, USA). Serum BAP was measured by the wheat germ lectin precipitation method (12). Serum PICP and PINP were measured by RIA (Orion Diagnostica, Espoo, Finland). Urinary levels of total Pyd and Dpd were measured by HPLC after acid hydrolysis (13). Urinary NTx was measured by ELISA (Osteomark, Ostex International, Inc., Seattle, WA). Urinary creatinine $(\mathrm{Cr})$ was measured in the Department of Clinical Chemistry at Northern General Hospital, Sheffield, using a dry slide chemistry autoanalyser (Johnson and Johnson, Ektachem 950). Urinary levels of Pyd, Dpd and NTx were expressed as a ratio to urinary level of Cr. The overall intra- and inter-assay coefficients of variation were 4 and $6 \%$ for IL- 6,7 and $10 \%$ for BGP, 3 and $5 \%$ for BAP, 6 and $8 \%$ for PICP, 6 and $8 \%$ for PINP, 2 and $4 \%$ for TRAP, 5 and $7 \%$ for NTx, 7 and $8 \%$ for Pyd and 9 and $10 \%$ for Dpd, respectively. The minimal detectable concentration of IL- 6 was determined to be less than $0.70 \mathrm{pg} / \mathrm{ml}$ by adding two standard deviations to the mean optical density value of 20 zero standard replicates and calculating the corresponding concentration. The assay recognises both natural and recombinant human IL-6 and no significant cross-reactivity with any factors related to or associated with IL- 6 has been observed.

Non-parametric statistical analysis was used in this study due to the small sample size. The Wilcoxon signed rank test was used to compare the difference between patients with PHPT and age- and sex-matched healthy controls at baseline. The repeated measures Friedman test with multiple comparison (Dunn's test, $P<0.05$ ) was used to compare the changes between different time points for patients with PHPT and surgical controls. Area under the curve (AUC) and Kruskal-Wallis tests were used to compare the percentage change between different biochemical markers of bone resorption after PTX. Spearman's rank correlation was used to examine the relationship between biochemical markers of bone turnover and serum IL- 6 at baseline.

\section{Results}

\section{Baseline}

Serum levels of IL-6, BGP, BAP, PINP, and urinary ratios of $\mathrm{Pyd} / \mathrm{Cr}, \mathrm{Dpd} / \mathrm{Cr}$ and $\mathrm{NTx} / \mathrm{Cr}$ were significantly higher PHPT than in healthy controls (Wilcoxon signed rank test $P=0.001,0.02,0.06,0.04,0.01,0.005$ and 0.007, respectively, Figs 1 and 2). The serum level of IL-6 did not correlate significantly with any biochemical marker of bone turnover.

\section{Follow-up}

Serum IL-6 levels increased immediately (by 1-2 h) with a maximum increase 1 day after PTX in PHPT, and had increased by 2 days after operation in surgical controls (repeated measures Friedman's test with Dunn's test at $P<0.05$, Fig. 3). 

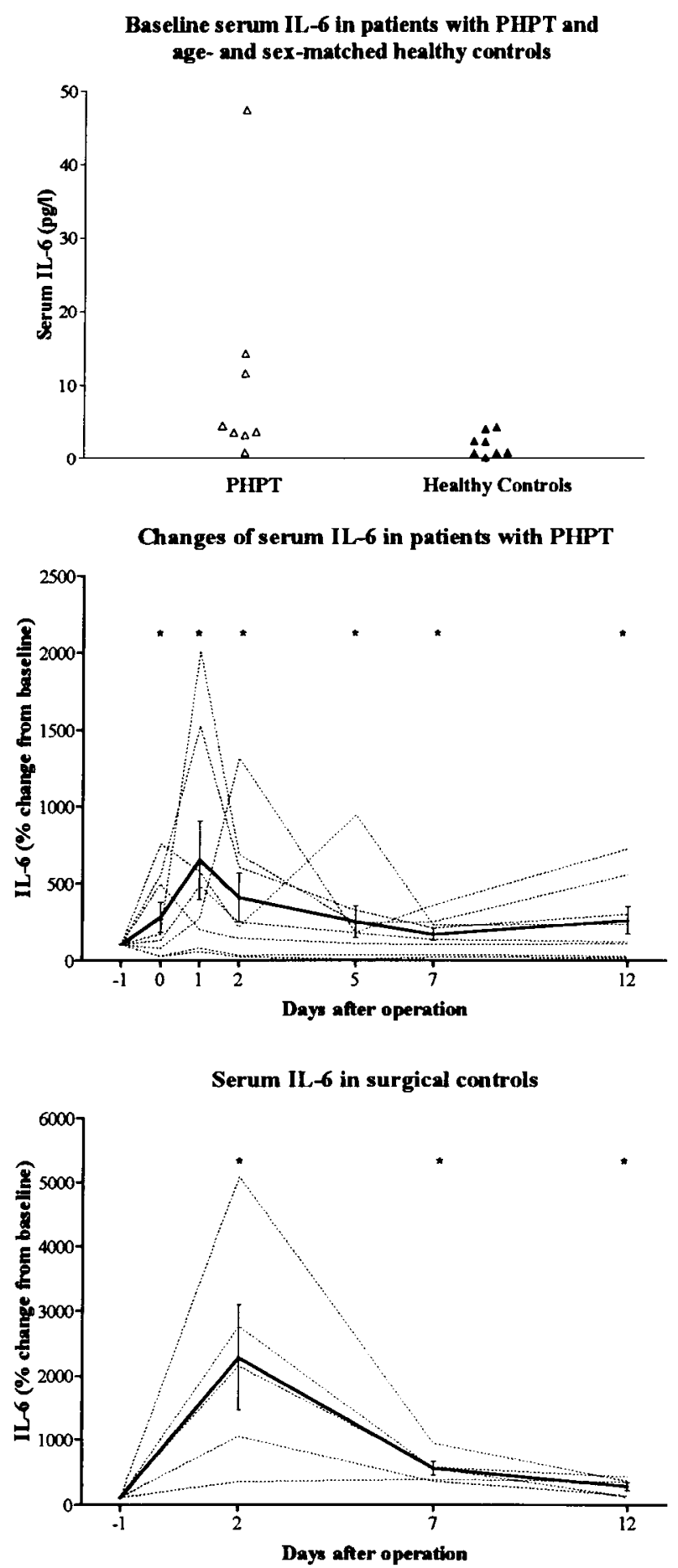

Figure 1 Serum IL-6 concentration at baseline in patients with PHPT and in age- and sex-matched controls (upper panel) and percentage changes after operation in patients with PHPT (middle panel) and surgical controls (lower panel). Asterisks indicate $P<0.05$. Solid lines represent mean change \pm S.E.M. Fine lines represent change for individual patients.
Serum levels of BGP and BAP decreased transiently from day 1 after PTX and then began to increase on days 5 and 12, respectively. Compared with baseline, serum levels of BGP and BAP did not change significantly even though there was a tendency to decrease within 5 days of PTX. Serum levels of PICP and PINP began to increase on day 2 and day 5 , respectively (repeated measures Friedman's test, Fig. 3). The maximum increase was found to occur on day 5 for PINP $(22 \%)$ and day 12 for PICP (27\%). Biochemical markers of bone formation did not change significantly in the five surgical controls during follow-up (repeated measures Friedman's test $P>0.05$, Fig. 4). However, serum levels of BGP and BAP showed a tendency to decrease, and PICP and PINP showed a tendency to increase.

There was no significant difference between biochemical markers of bone resorption measured in healthy controls and these markers measured at any time in patients with PHPT after PTX. Compared with baseline, serum TRAP decreased significantly from day 2 after PTX whereas urinary $\mathrm{Pyd} / \mathrm{Cr}$, Dpd/Cr and NTx/Cr decreased significantly from day 1 after PTX (repeated measures Friedman's teest, Fig. 5). The maximum decrease was found to occur on day 7 (21\%) for serum TRAP and on day 1 for urinary Pyd/Cr (21\%), $\mathrm{Dpd} / \mathrm{Cr}(33 \%)$ and NTx/Cr (41\%) after PTX. Serum TRAP did not change significantly in the surgical controls (not shown). We did not find significant differences in postoperative change (AUC) between serum TRAP, urinary $\mathrm{Pyr} / \mathrm{Cr}$, Dpyr/Cr and NTx/Cr after PTX in patients with PHPT (one-way ANOVA $P>0.05)$.

\section{Discussion}

PTH infusion studies have shown that PTH increases bone resorption (1-4) and decreases bone formation (4-6). Therefore, a decreased bone resorption and an increased bone formation would be expected shortly after PTX. The interesting finding in the present study was that all collagen-derived markers of bone resorption decreased and normalised immediately within $24 \mathrm{~h}$ whereas serum levels of BAP and BGP did not change significantly after PTX, which contrasts with PTH infusion studies and some previous PTX studies $(14,15)$ but not all $(16-20)$.

Indeed, serum levels of BGP and BAP decreased slightly shortly after PTX in the present study but similar changes were found in the surgical controls. This could be explained by the stress of surgery. Elevated levels of serum cortisol have been reported for up to 3 days after major abdominal surgery $(21,22)$. Serum levels of PICP and PINP increased significantly in PHPT after PTX in the present study. However, serum levels of PICP and PINP also showed a tendency to increase in surgical controls. Following major abdominal surgery, increased procollagen synthesis has been found within 2-4 days at the site of the wound 


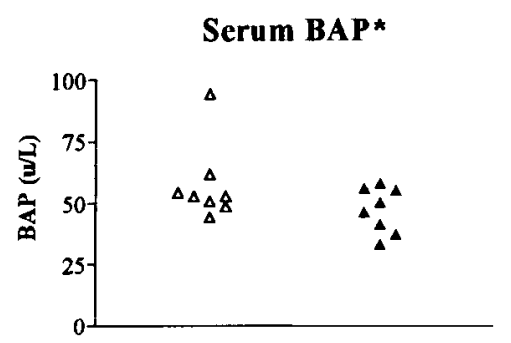

Serum PINP *

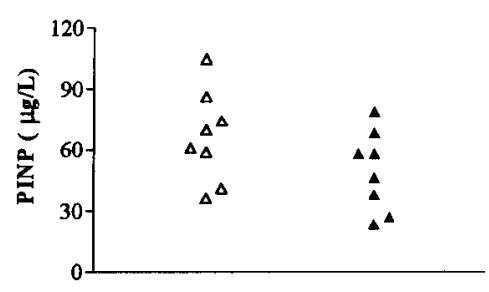

Serum TRAP

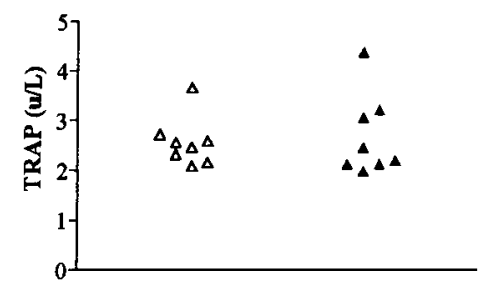

Urinary $\mathrm{NT} x / \mathrm{Cr}^{*}$

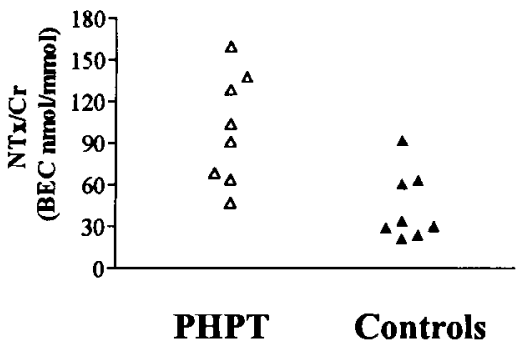

(23-25), and serum PICP started to increase from day 4 (25) with a maximum increase occurring between days 10 and $21(26,27)$. Serum PICP has been reported to be increased $3-5$ days after PTX $(18,19)$ which is consistent with the present study. In the present study, both PICP and PINP increased about 20\% from 5 to 7 days after surgery in surgical controls. This result suggests that in PHPT after PTX, the increased procollagen synthesis in the early phase represents increased bone matrix synthesis whereas in the late phase represents wound healing.

Urinary levels of $\mathrm{Pyd} / \mathrm{Cr}, \mathrm{Dpd} / \mathrm{Cr}$ and $\mathrm{NTx} / \mathrm{Cr}$ decreased significantly within $24 \mathrm{~h}$ after PTX. The immediate decrease in bone resorption cannot be explained by a slowing down of osteoclast recruitment which would last days or weeks. The rapid decrease in bone resorption could be explained by the immediate release of existing osteoclasts from their stimulation by PTH. It has been suggested that PTH acts later in the osteoclast lineage rather than in the formation of new osteoclasts (10). This finding also supports the hypothesis that PTH acts on the osteoclast itself, although this is controversial (10).

Garton and colleagues (15) showed that urinary Dpd decreased by $48 \mathrm{~h}$ after PTX in ten patients (no samples were taken within $24 \mathrm{~h}$ after PTX in that study) whereas urinary Pyd did not change significantly during 6 months follow-up after PTX. Urinary Pyd has been reported to be less specific to bone than urinary Dpd since urinary Pyd may originate from 
Serum BAP

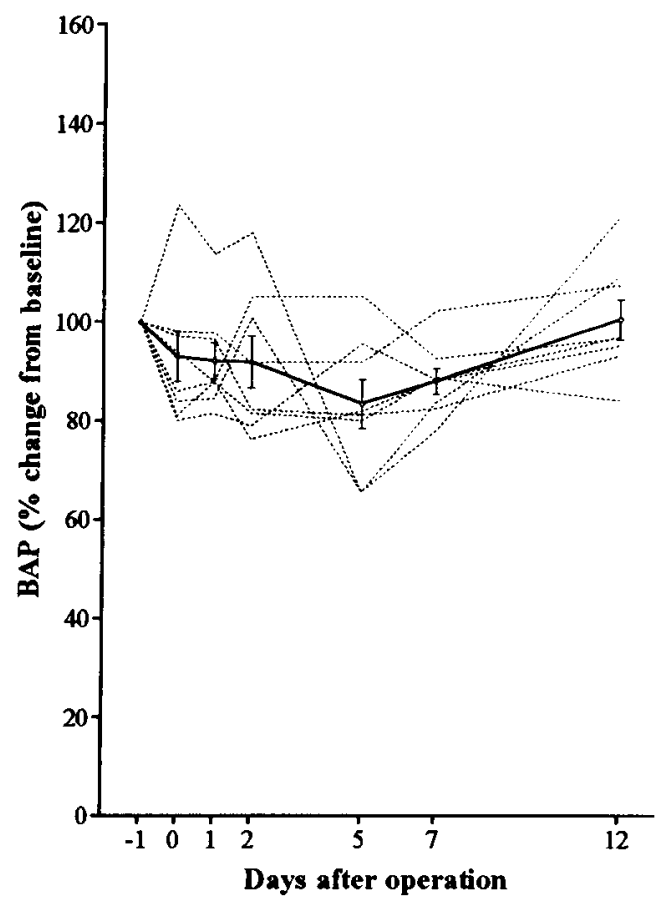

Serum PINP

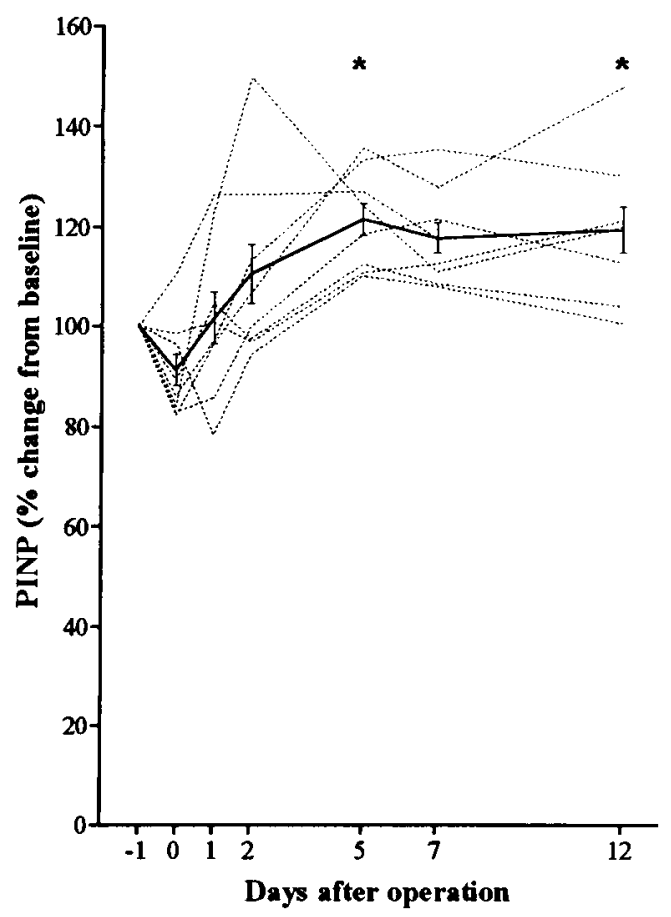

Serum BGP

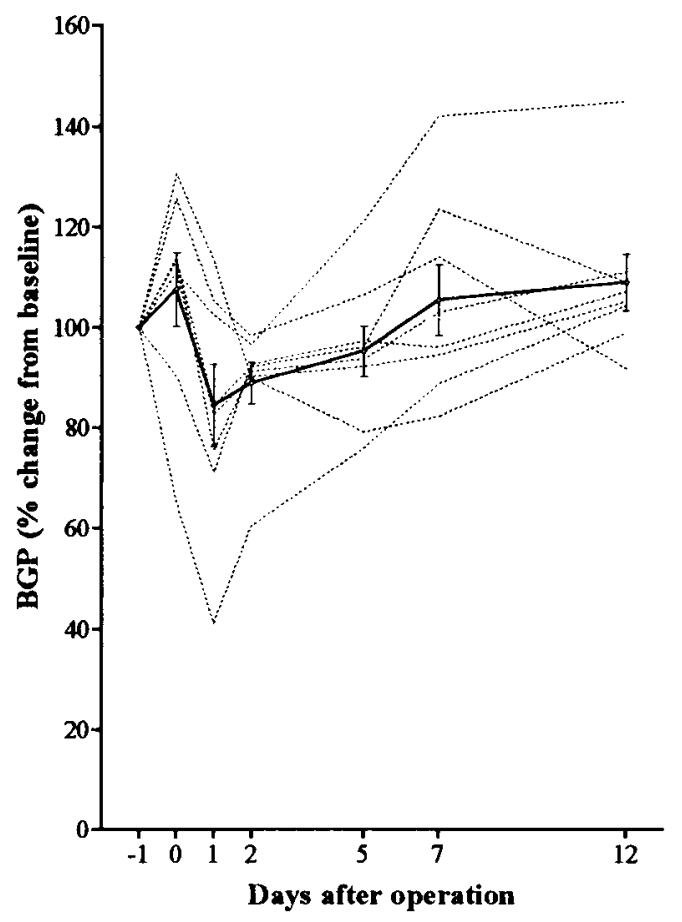

Serum PICP

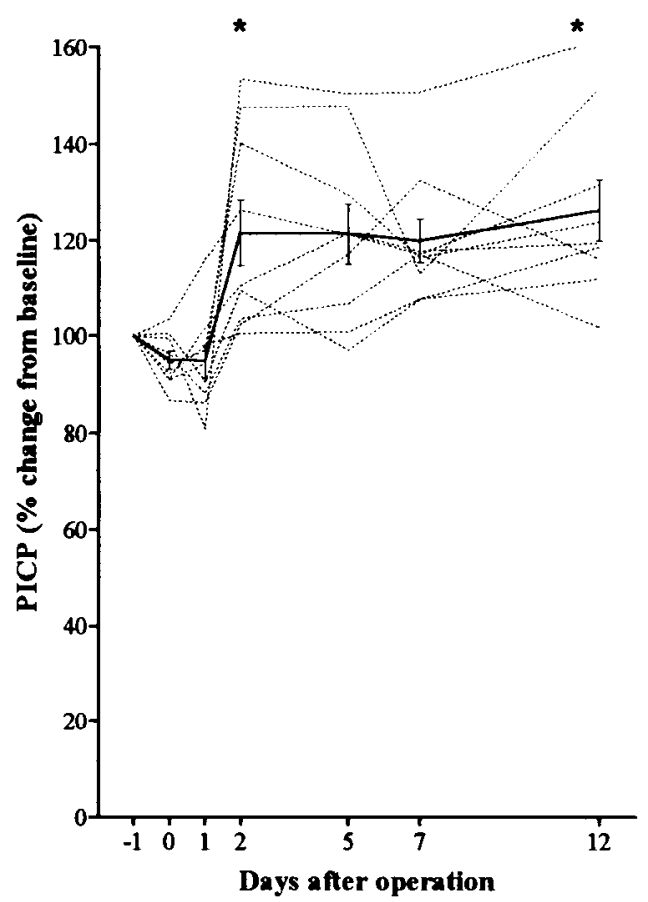

Figure 3 Changes in biochemical markers of bone formation in patients with PHPT before and after PTX. Asterisks indicate $P<0.05$ compared with baseline. 
Serum BAP

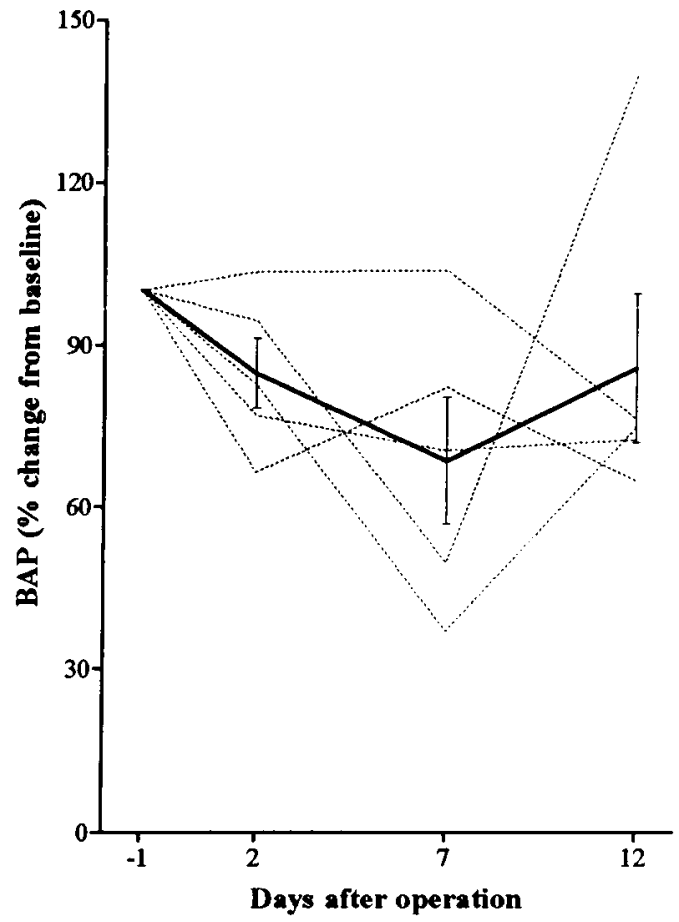

Serum PINP

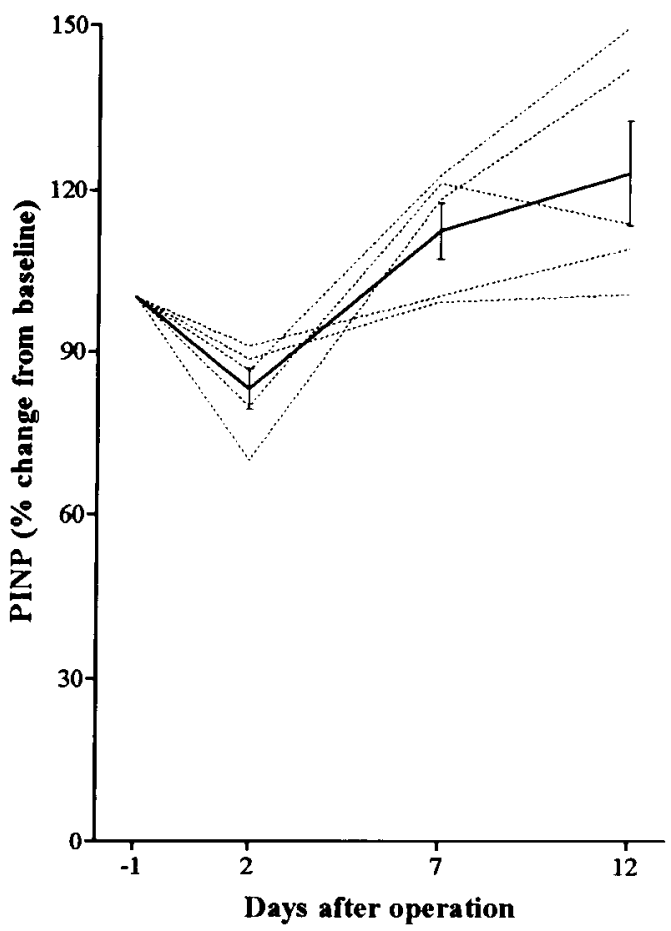

Serum BGP

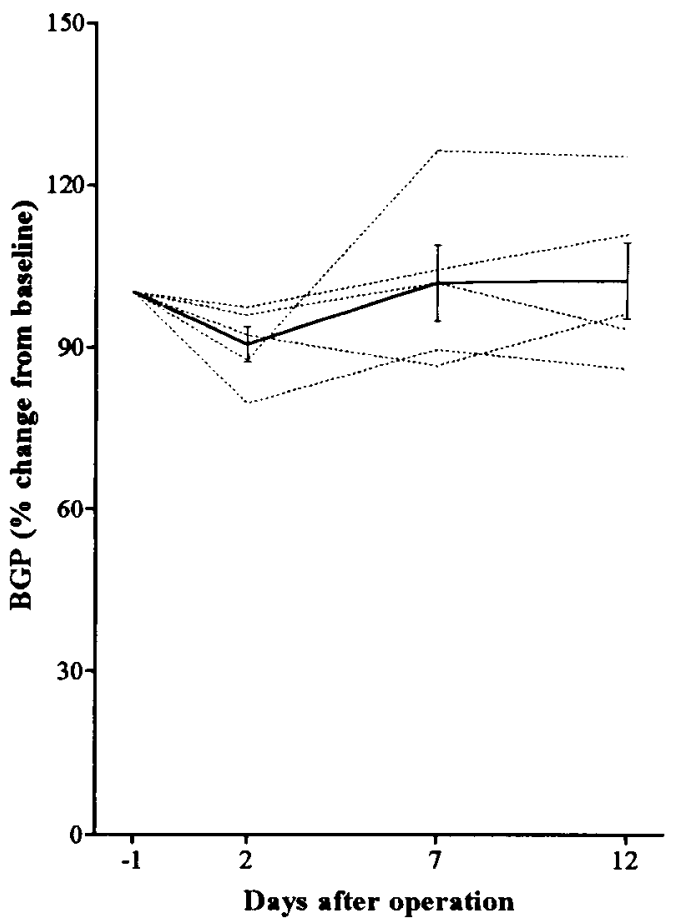

Serum PICP

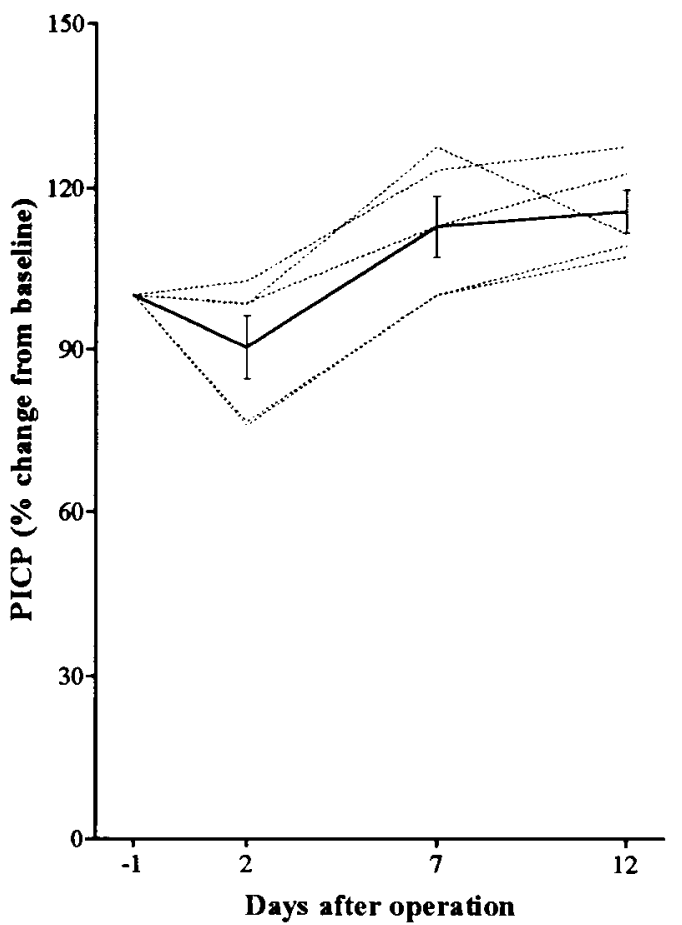

Figure 4 Changes in biochemical markers of bone formation in surgical controls before and after surgery. Asterisks indicate $P<0.05$ compared with baseline. 
Serum TRAP

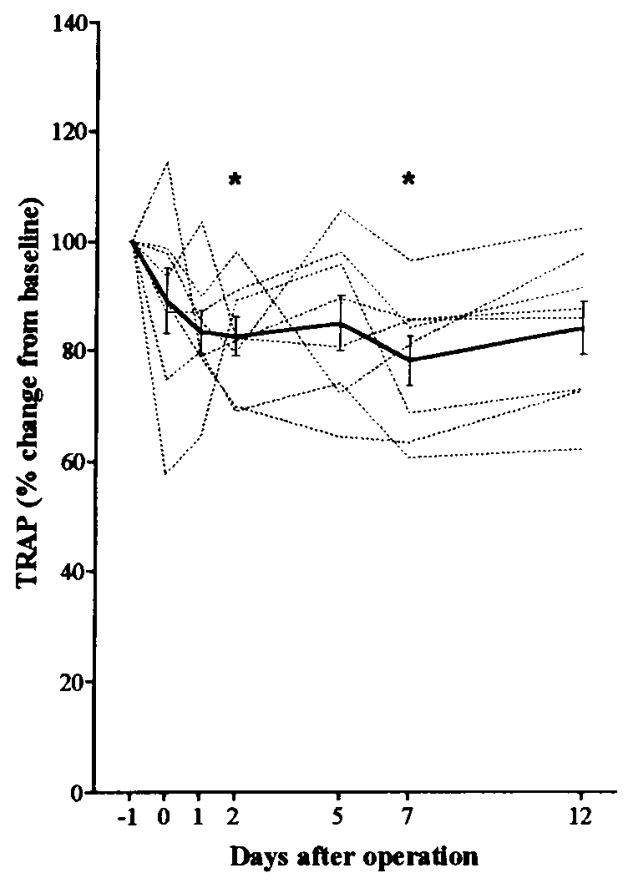

Urinary NTx/Cr

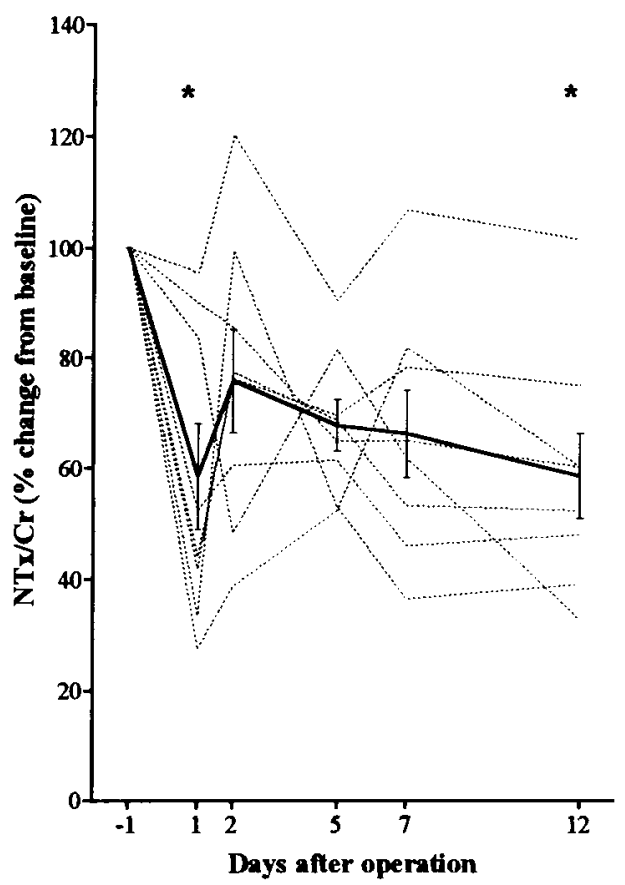

Urinary Pyd/Cr

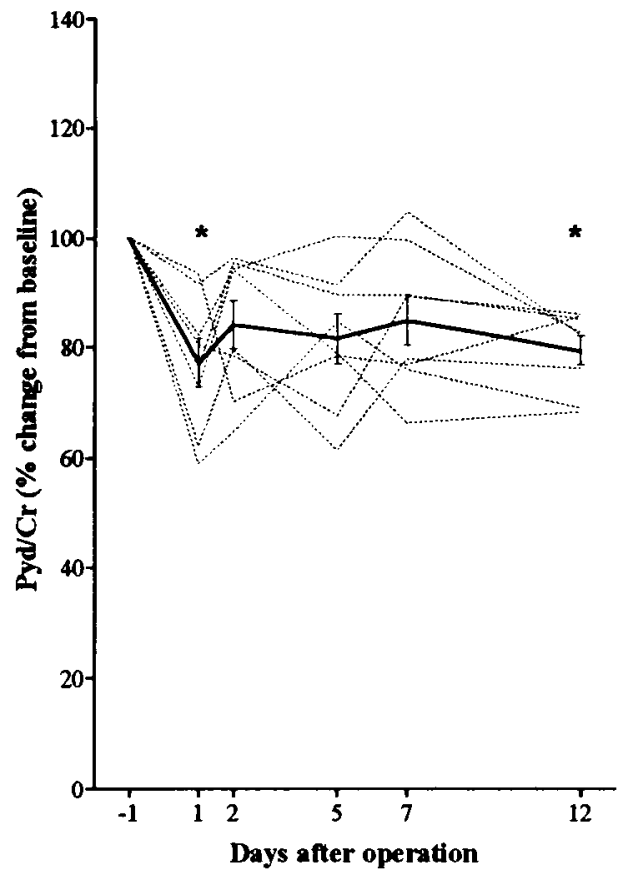

Uninary Dpd/Cr

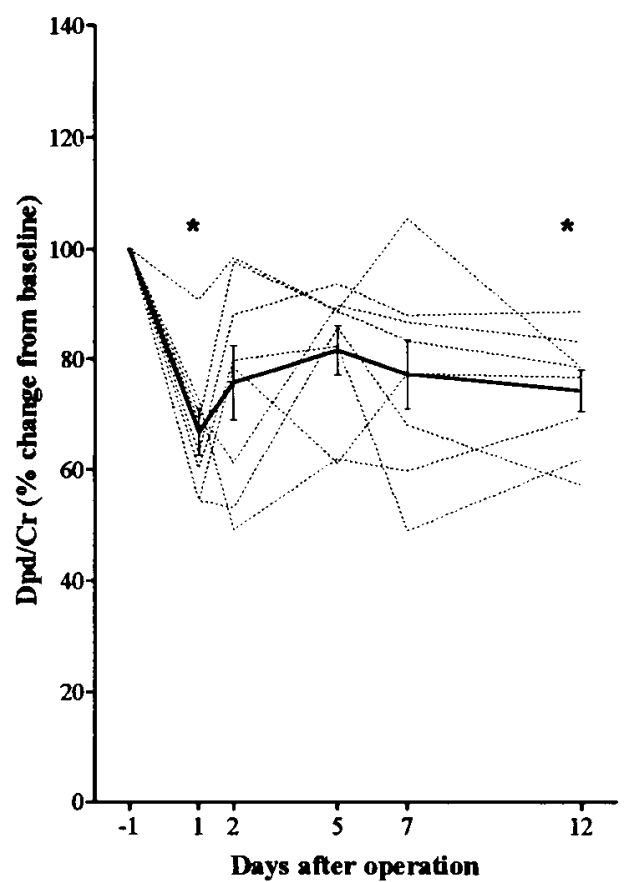

Figure 5 Changes in biochemical markers of bone resorption in patients with PHPT before and after PTX. Asterisks indicate $P<0.05$ compared with baseline. 
tissues containing type II collagen such as cartilage. Previous studies reported that the maximum decrease in serum TRAP level occurred between 5 and 10 days after PTX in patients with PHPT $(16,19)$. This is consistent with the present study. Serum TRAP as an enzyme released from osteoclasts represents osteoclast numbers whereas pyridinium crosslinks of collagen represent osteoclast function. Osteoclast apoptosis may take a few days. Another possibility is that the half-life for TRAP in serum may be longer than that for crosslinks.

It is likely that most of the decrease in collagen degradation occurs in the first postoperative day. We found a decrease of $41 \%$ in NTx/Cr on day 1 , similar to the $52 \%$ decrease we reported previously for up to 20 months follow-up after PTX (7). In a study with up to 24 months follow-up after PTX, Seibel and colleagues (28) reported that although urinary Dpd decreased by $37 \%$ in $0.5-6$ months after PTX, a further decrease (up to $53 \%$ ) was found in 7-12 months after PTX compared with baseline. Nevertheless, our results showed that significant decrease of bone resorption occurred within $24 \mathrm{~h}$ even though a further small decrease in long-term follow-up may occur.

PTH receptors in bone cells are found only in osteoblasts or osteoblast precursors and the mechanisms of the effect of PTH on osteoclasts are generally considered to be mediated by cytokines such as IL-6 (10). A recent study has reported that serum levels of IL-6 and IL-6 soluble receptors were increased significantly in untreated patients with PHPT and fell into the normal range after surgery (11). We confirmed high baseline IL-6 levels in PHPT. If IL-6 were the main determinant of bone resorption, we would have expected a immediate decrease of serum IL-6 after PTX. However, the effects of PTH on bone resorption are unlikely to be mediated by IL- 6 since serum levels of IL- 6 increased in both patients with PHPT and surgical controls after surgery. This result indicated that the increased IL- 6 concentration was not related to bone turnover directly. The concentration of IL-6 in serum and plasma has been shown to increase in response to surgery and has been proposed as a sensitive marker of tissue damage (29-31). Since local IL-6 levels in bone may not parallel circulating IL-6 levels, we cannot entirely rule out the possibility that the increased circulating IL- 6 level induced by surgical trauma masks a decrease in bone IL-6 levels induced by a decrease in PTH level.

We conclude that (1) PTX results in an immediate decrease in bone resorption and (2) circulating IL-6 is not involved in the changes of bone turnover immediately after PTX.

\section{Acknowledgements}

The authors thank Ostex International Inc. (Seattle, WA, USA), for the NTx kits supplied free of charge.

\section{References}

1 Cosman F, Shen V, Herrington B \& Lindsay R. Response of the parathyroid gland to infusion of human parathyroid hormone(1-34) (PTH-(1-34)): Demonstration of suppression of endogenous secretion using immunoradiometric intact PTH-(1-84) assay. Journal of Clinical Endocrinology and Metabolism 199173 1345-1351.

2 Tsai KS, Ebeling PR \& Riggs BL. Bone responsiveness to parathyroid hormone in normal and osteoporotic postmenopausal women. Journal of Clinical Endocrinology and Metabolism 198969 1024-1027.

3 Hodsman $\mathrm{AB}$ \& Fraher LJ. Biochemical responses to sequential human parathyroid hormone (1-38) and calcitonin in osteoporotic patients. Bone and Mineral 19909 137-152.

4 Joborn C, Ljunghall S, Larsson K, Lindh E, Naessen T, Wide L, Akerstrom G \& Rasted J. Skeletal responsiveness to parathyroid hormone in healthy females: relationship to menopause and oestrogen replacement. Clinical Endocrinology 199134 335-339.

5 Simon, LS, Slovik DM, Neer RM \& Krane SM. Changes in serum levels of type I and III procollagen extension peptides during infusion of human parathyroid hormone fragment (1-34). Journal of Bone and Mineral Research 19883 241-246.

6 Brahm H, Ljunggren O, Larsson K, Lindh E \& Ljunghall S. Effects of infusion of parathyroid hormone and primary hyperparathyroidism on formation and breakdown of type I collagen. Calcified Tissue International 199455 412-416.

7 Guo C-Y, Thomas WEG, Al-Dehaimi AW, Assiri AMA \& Eastell R. Longitudinal changes in bone mineral density and bone turnover in postmenopausal women with primary hyperparathyroidism. Journal of Clinical Endocrinology and Metabolism $1996 \quad 81$ 3487-3491.

8 Grey AB, Stapleton JP, Evans MC \& Reid IR. Accelerated bone loss in postmenopausal women with primary hyperparathyroidism. Clinical Endocrinology 199644 697-702.

9 Silverberg SJ, Gartenberg F \& Jacobs TP. Longitudinal measurements of bone density and biochemical indices in untreated primary hyperparathyroidism. Journal of Clinical Endocrinology and Metabolism $199580723-728$.

10 Mundy GR. Bone remodelling and its disorders, pp 39-41. London: Martin Dunitz 1995.

11 Grey A, Mitnick MA, Shapses S, Ellison A, Gundberg C \& Insogna K. Circulating levels of interleukin- 6 and tumor necrosis factor-a are elevated in primary hyperparathyroidism and correlate with markers of bone resorption - a clinical research center study. Journal of Clinical Endocrinology and Metabolism 1996 $813450-3454$.

12 Rosalki SB \& Foo AY. Two new methods for separating and quantifying bone and liver alkaline phosphatase isoenzymes in plasma. Clinical Chemistry $1984301182-1186$.

13 Colwell A, Russell RGG \& Eastell R. Factors affecting the assay of urinary 3-hydroxypyridinium crosslinks of collagen as markers of bone resorption. European Journal of Clinical Investigation 1993 23 341-349.

14 Minisola S, Scarnecchia L, Scarda A, Bigi F, Tabolli S, Valtorta C \& Mazzuoli G. Serum osteocalcin in primary hyperparathyroidism: short-term effect of surgery. Mineral Electrolyte Metabolism $198814201-207$.

15 Garton M, Martin J, Stewart A, Krukowski Z, Matheson N, Robins S, Loveridge $\mathrm{N} \&$ Reid D. Change in bone mass and metabolism after surgery for primary hyperparathyroidism. Clinical Endocrinology 199542 493-500.

16 Stepan JJ, Silinkova-Malkova E, Havranek T, Formankova J, Zichova M, Lachmanova J, Strakova M, Broulik P \& Pacovsky V. Relationship of plasma tartrate resistant acid phosphatase to the bone isoenzyme of serum alkaline phosphatase in hyperparathyroidism. Clinica et Chimica Acta 1983133 189-200.

17 Delmas PD, Demiaux B, Malaval L, Chapuy MC, Edouard C \& Meunier PJ. Serum bone gamma carboxyglutamic acid-containing 
protein in primary hyperparathyroidism and in malignant hypercalcemia. Comparison with bone histomorphometry. Journal of Clinical Investigation 198677 985-991.

18 Coen G, Mazzaferro S, De Antoni E, Chicca S, DiSanza P, Onorato L, Spurio A, Sardella D, Trombetta M, Manni M \& Pasquali M. Procollagen type I C-terminal extension peptide serum levels following parathyroidectomy in hyperparathyroid patients. American Journal of Nephrology 199414 106-112.

19 Minisola S, Romagnoli E, Scarnecchia L, Rosso R, Pacitti MT, Scarda A \& Mazzuoli G. Serum carboxy-terminal propeptide of human type I procollagen in patients with primary hyperparathyroidism: studies in basal conditions and after parathyroid surgery. European Journal of Endocrinology 1994130 587-591.

20 Casey MC, Iqbal SJ, Bell PRF \& Lunec J. Advantages of newer bone markers to traditional biochemistry in pre- and post-operative primary hyperparathyroidism: a one year follow-up study. Journal of Endocrinology 1996148 (Suppl) 187.

21 Naito Y, Tamai S, Shingu K, Shindo K, Matsui T, Segawa H,

Nakai Y \& Mori K. Responses of plasma adrenocorticotropic hormone, cortisol, and cytokines during and after upper abdominal surgery. Anesthesiology 199277 426-431.

22 McIntosh TK, Lothrop DA, Lee A, Jackson BT, Nabseth D \& Egdahl RH. Circadian rhythm of cortisol is altered in postsurgical patients. Journal of Clinical Endocrinology and Metabolism 198153 117-122.

23 Haukipuro K, Melkko J, Risteli L, Kairaluoma M \& Risteli J. Synthesis of type I collagen in healing wounds in humans. Annual of Surgery 1991213 75-80.

24 Haukipuro K. Synthesis of collagen types I and III in reincised wounds in human. British Journal of Surgery 199178 708-712.
25 Ihlberg L, Haukipuro K, Risteli L, Oikarinen A, Kairaluoma MI \& Risteli J. Collagen synthesis in intact skin is suppressed during wound healing. Annals of Surgery 1993217 397-403.

26 Haukipuro K, Risteli L, Leppilahti J, Melkko J, Puranen J, Kairaluoma MI \& Risteli J. Responses of type I and type III collagen synthesis after replacement of the hip joint. Journal of Surgical Research 199558 443-448.

27 Haukipuro K, Melkko J, Risteli, L, Kairaluoma MI \& Risteli J. Connective tissue response to major surgery and postoperative infection. European Journal of Clinical Investigation 199222 333-340.

28 Seibel M, Gartenberg F, Silvergerg S, Ratcliffe A, Robins SP \& Bilezikian JP. Urinary hydroxypyridinium cross-links of collagen in primary hyperparathyroidism. Journal of Clinical Endocrinology and Metabolism 199274 481-486.

29 Shenkin A, Fraser WD \& Series J. The serum interleukin-6 response to elective surgery. Lymphokine Research 19898 123-127.

30 Cruickshank AM, Fraser WD, Burns HJG, van Damme J \& Shenkin A. Response of serum interleukin- 6 in patients undergoing elective surgery of varying severity. Clinical Science 199079 161-165.

31 Kristiansson M, Soop M, Saraste L \& Sundqvist KG. Post-operative circulating cytokine patterns - the influence of infection. Intensive Care Medicine 199319 395-400.

Received 14 July 1999

Accepted 27 January 2000 